\title{
EXPERIMENTS AND OBSERVATIONS
}

ON THS

\section{SERUM OF THE BLOOD,}

BY JOHN BOSTOCK, M.D.

OF LIVERPOOL.

Read April 23, 1811.

IN a paper which was published in the Transactions of the Medical and Chirurgical Society of London*, I maintained the opinion that blood does not contain any constituent to which the name of jelly or gelatine can be properly applied. 'As this opinion was in opposition to the highest chemical authorities $t, I$ could not expect it to be received without opposition, more especially as the experiments which were adduced in its favour, were both few in number, and very simple in their nature. Since that time I have examined the subject with more attention, and I have endeavoured to vary the experiments in such a way, as to meet the objections to which the former were liable. It may be necessary to premise, that by the term

* Medico-Chirurgical Trans. Vol. I. p. 47.

$\uparrow$ See the references in the paper referred to, p. 69. 
jelly, I mean that animal substance which possesses the characteristic property of being liquefied by heat, and becoming concrete by cold, and which forms with $\tan$ a compound that is insoluble in water.

By the modern authors who have written upon the blood, it is asserted, that when serum is coagulated by heat, the albuminous part alone is rendered solid, and that there may be separated from the coagulated albumen a fluid, which holds a quantity of jelly in solution. To this fluid, which appears first to have been distinctly recognized by Dr. Butt, Cullen restricted the term of serosity, which had been previously applied by Senac to the serum at large; MM. Fourcroy and Vauquelin first announced the existence of jelly in it, MM. Parmentier and Deyeux further developed the sup. posed discovery, and since that time its existence has never been called in question. The serosity may be procured either by permitting it to ooze spontaneously from coagulated serum cut into small pieces, or more readily, by digesting the coagulated serum in boiling water. If the quan. tity of water employed be not more than the bulk of the serum, the fluid is very nearly the same as obtained by either of these means ; for, in consequence of the serosity being lodged in minute cells dispersed through the serum, which are penetrated by the boiling water, it is by this operation more completely separated from the albumen. The solid 
contents of the serosity generally amount to about $\frac{1}{50}$ of the weight of the fluid, but in different specimens I have found them to vary from $\frac{1}{46}$ to $\frac{1}{70}$. It is slightly opake, its taste is saline, and it has an odour which has always appeared to me specific, and different from that of serum; it is generally neither acid or alkaline, but occasionally I have thought that I could perceive in it the slightest degree of alkalescency *. When the serosity has the oxymuriate of mercury added to it, or when it is subjected to the heat of boiling water, no effect is produced, and I never could perceive that the slight degree of opacity which it naturally possesses was increased; but when the two operations are united, when the oxymuriate of mercury is added, and the mixture is kept for some time at the boiling temperature, there is a manifest increase of opacity, and a precipitate is formed. An infusion of tan always increases the opacity of the serosity, and a precipitate very gradually subsides from it $t$. When serosity has been exposed to the atmosphere for

- The alkaline test which I generally employ is turmeric; it possesses the advantage of not being injured by keeping, and it is obviously affected by a solution containing $\frac{1}{5000}$ of its weight of potash.

The preparation of $\tan$ which I have found the most convenient for the experiments, is formed by the extract of rhatany. If the rhatany be digested in hot water, and filtered after it becomes cold, a solution is formed of nearly uniform strength, and which remains a considerable time without moulding or undergoing any spontaneous change. In both these respects it is superior either to catechu or to galls. 
some days, it becomes more opake, a flaky substance separates from it, and its odour is extremely nauseous : in this state it exhibits an excess of alkali. I have never been able to detect any degree of acidity during its spontaneous decomposition, although this has been asserted to be the case * When the serosity is evaporated by a gentle heat, it gradually becomes less clear, small flakes are formed in it, and at length a semitransparent substance is left, of a membranous appearance, which evidently consists, at least for the most part, of animal matter. By exposure to a greater heat, it grows brown, emits a peculiar smell, and at length becomes charred. If the serosity, after being evaporated to dryness; be digested in boiling water, it is re-dissolved, except a small quantity of flaky matter, which floats in it, and which seems incapable of solution. When the solid contents of the serosity, after having been evaporated, and reduced to a half charred state, are digested in boiling water, the fluid is rendered brown, and upon evaporation a brown mass is left, which contains small cubical crystals of the muriate of soda $t$. If

* I suspect the generation of acid in serosity must be classed among those numerous occurrences, where the experimentalist has been guided more by hypothesis than by observation. When serosity had been announced to be a gelatinous fluid, it followed of course that it must evolve an acid during its spontaneous decom-' position. See Brugmans as quoted in Thompson's Fourcroy, III. 272.

$\uparrow$ It was always supposed that the muriatic acid in serum was combined with soda, until Dr. Pearson lately endeavoured to 
the evaporation of the serosity be stopped during the process, and the substance cooled, it does not exhibit any appearance of gelatinization. Acetate of lead and nitrate of silver both throw down copious precipitates from the serosity.

From these experiments we may conclude, that serosity consists of water holding in solution nearly उo of its weight of an animal substance, together with a small quantity of the muriate of soda. Of this animal matter a portion is albumen; the exact quantity cannot be ascertained, because it is not practicable to collect the precipitate which is occasioned by boiling the serosity with the oxymuriate of mercury; but from comparing the effects with what takes place, when a known quantity of albumen ovi is added to water, we may conclude, that the serosity cannot contain more than $\frac{1}{1000}$ of its weight of albumen. That the remaining animal matter in serosity is not jelly, may be at once inferred from the consideration, that if water contains only $\frac{1}{10} \overline{0}$ of its weight of jelly, it becomes concrete at the usual temperature of the atmosphere, whereas the serosity is perfectly fluid, although the animal matter in it is not much less than double

prove it to be potash. My experiments induce me to adhere to the ofd opinion, as I never found the cubical crystals mentioned above to praduce any precipitate with the nitro-muriate of platina. The addition of oxalic acid to serum shews that it contains a minute quantity-of lime, and it is sajd that the phosphate of soda also exists in it. 
that quantity. In order to ascertain whether there was any portion of jelly in serosity, the following experiments were tried. To a quantity of serum $\frac{1}{10}$ of its weight of jelly, liquefied by heat, was added, and upon cooling the mixture, it became quite concrete. To $94 \mathrm{grs}$. of serosity, $6 \mathrm{grs}$. of a solution of jelly, containing $\frac{1}{20}$ of a grain of solid jelly, was added, so that the jelly would form $\frac{1}{2000}$ part of the weight of the whole fluid. This was exposed to a gentle heat, and when it was nearly evaporated, upon being cooled, a very obvious degree of gelatinization took place. One fact still remained to be explained, which seemed to be the only one that opposed my conclusion, and this was the effect of $\tan$ on the serosity. The precipitate that is produced when tan is added to serosity, has always been supposed to indicate the presence of jelly*; but tan, although it dơes not detect albumen as minutely as it does jelly, yet it produces an obvious effect in a fluid that contains

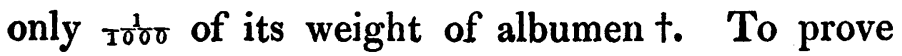

- In the most recent analysis of animal fluids, it has been as:sumed as a sufficient proof of the presence of jelly, that a precipitate is produced by tan. From this circumstance alone M. Nicolas concludes that the aqueous humor of the eye contains jelly. Ann. Chim. T. LIII. p. 310.

+ If to a quantity of water $\frac{1}{12} \sigma$ of its bulk of serum be added, we shall have a mixture which contains about $\frac{1}{10 \delta \delta}$ of its weight of solid albumen. Equal parts of this mixture and the infusion of rhatany being added together, produce an immediate and very perceptible precipitation. 
that the action of tan on serosity depends upon the small quantity of albumen still remaining in it, a portion of serum was diluted with 4 times its bulk of water, to this was added $\frac{1}{8}$ of its bulk of a saturated solution of the oxymuriate of mercury, and the mixture was kept for some time at the boiling temperature; a hard dense precipitate was formed, the fluid was left transparent, and now it produced no precipitate with the solution of tan. Against this experiment it might be urged, that although the oxymuriate of mercury has no action upon jelly alone, yet that when a small quantity of jelly is united to a large proportion of albumen, the compound may be precipitated by the oxymuriate. To examine how far this objection was valid, I added to a portion of serum about ${ }_{T}^{\frac{1}{0} \sigma}$ of its weight of jelly, and then boiled it with the oxymuriate of mercury. ' A precipitate was formed of the usual appearance; but as the fluid cooled, the jelly formed a distinct concretion, and did not appear to have been incorporated with the albumen, or affected by the oxymuriate of mercury. There was another obvious difference between the precipitates formed by tan in serosity and in jelly; the precipitate in the serosity is incoherent, it subsides very slowly, and can scarcely be separated from the fluid by a filtre, whereas the precipitate in a solution of jelly of the same strength is a hard dense substance, which almost immediately separates from the fluid, and may be collected in a distinct mass. 
Conceiving it to be sufficiently established, that there is no jelly in serosity, the next object of inquiry was, whether the opinion be correct, that only a small part of the animal matter in it is albumen. For this purpose I examined the effects produced by the same re-agents upon serosity, and upon serum diluted with water, until its solid contents were equal, or even inferior in quantity to those of the serosity. A quantity of serum, which contained twelve per cent. of solid matter, was diluted with eleven times its bulk of water, so that its solid contents would be considerably less than that of the serosity, and the following comparative experiments were then performed. A boiling heat produced in the diluted serum a perceptible opacity, but in the serosity no effect. The same quantity of oxymuriate of mercury was added to both these fluids, it produced an evident precipitate in the diluted serum, but none in the serosity; when the boiling heat was applied to the two portions of fluid containing the oxymuriate of mercury, it produced in the diluted serum a distinct precipitate, which separated from the fluid and subsided; in the serosity there was a degree of opacity produced, but no distinct precipitate was formed. Even when this serum was still farther diluted, with three times its bulk of water, so that its solid contents could not have been $\frac{1}{6}$ as much as those of the serosity, the joint effects of heat and the oxymuriate of mercury caused a greater degree of opaeity. And there is a still more decided fact in favour of my 
opinion. If serum be boiled with such a quantity of the oxymuriate of mercury as to remove all the albumen, and to leave a slight excess of the oxymuriate, the least addition - of albumen will cause a fresh precipitate; yet if the fluid be evaporated, a residuum is procured, which is evidently in part animal matter. I have noticed a difference between albumen and the animal matter in serosity with respect to their solubility in water. Coagulated albumen is perfectly insoluble, as is proved by the constitution of the serum, and if albumen be very slowly evaporated by a heat not sufficient to coagulate it, it is capable of being redissolved, whereas if it be dried by a heat in any considerable degree greater than that necessary for coagulation, it remains insoluble in water. As far as $I$ have been able to ascertain the fact, with respect to the animal matter in serosity, it does not possess the property of being equally insoluble by being exposed to the same degree of heat. When serosity has been evaporated, the whole is not again soluble; but the insoluble portion I attribute to some remains of albumen, which we have been led, from other circumstances, to conclude still exists in it. The greater part however is soluble, and remains so even when the heat applied has been considerable enough to produce a commencement of carbonization. These facts I consider sufficient to warrant the conclusion, that the greatest part of the animal matter in serosity is not albumen. I shall not at present inquire into its properties, or 
into the denomination which ought to be applied to it, as I propose to enter more fully into this investigation on some future opportunity. I shall conclude this paper with some detached observations respecting the serum.

The specific gravity of serum is generally stated at 1.027 or $1.028^{*}$, the original authority for which appears to have been Jurin; I have examined several specimens, and have always found it to be less; the average of my experiments is about 1.023. The proportion of the solid contents of the serum to the watery part, varies less than might have been supposed, from the varieties that we observe in the state of the blood. I have generally found the solid contents left by a slow evaporation to be pretty exactly 12 per cent.

Serum always exhibits marks of an uncombined alkali, the amount of which $I$ have endeavoured to ascertain. After observing the effect that was produced by serum upon turmeric paper, I added a solution of potash, drop by drop, to a quantity of water, until it seemed to tinge the paper in an equal degree, when it was found that about ${ }_{10}^{1} \sigma 0$ of its weight of the solution had been added to the water. The strength of the alkaline solution was such, that it was saturated by half its weight of sul-

* See the Systems of Thompson and Murray, Henry's Epi. tome, and Aikin's and Parr's Dictionary. 
phuric acid of the specific gravity of 1.76. To 240 grains of serum acetic acid was added, until there was a very slight excess of acidity; then to an equal bulk of water the same proportion of the alkaline solution was added, as was indicated by the last experiment, when it was found, that as much acetic acid was necessary to saturate this water as the 240 grains of serum. From these two experiments it appeared, that one ounce of the serum required for its saturation, as nearly as possible, one grain of the sulphuric acid. Acetic acid, 100 parts of which require for saturation 43 parts of pure potash, will cause an evident excess of acidity in 60 times its bulk of serum.

It is generally supposed that the alkali in serum is in the caustic state, and the following experiments seem to favour this opinion; although it must be acknowledged, that the smallness of the quantity renders it difficult to speak with absolute certainty upon the subject. To a quantity of serum acetic acid was added, until there was a slight excess of acidity, but no effervescence could be observed. To another portion of serum, about T10 of its weight of a solution of a carbonated alkali was added, and this was then supersaturated with acetic acid, when a number of very minute bubbles might be discerned rising through the fluid. A solution of jelly was formed of about the same degree of viscidity with serum, and to this a solution of the carbonate of potash was added, so as to 
make it equally alkaline; when this was neutralized by acetic acid, an obvious effervescence was excited. As far as I could come to any certain conclusion respecting a point of so much minuteness, it did not appear that the alkali of serum became carbonated by being exposed to the atmosphere for a considerable length of time. Notwithstanding the alkalescency of the serum, the serosity scarcely ever exhibits any decisive marks of an uncombined alkali *.

In the experiments upon the serosity I have assumed, that its solid contents principally consist of animal matter ; there are, however, some saline substances in it, and it was important to ascertain the proportion which they bore to the whole. For this purpose a quantity of coagulated serum was cut into small pieces, and boiled with successive portions of water, until all the soluble matter seemed to be separated from it; these different portions of water were then evaporated, and the residuum was dried by a heat sufficient to reduce it to a half charred state. It was then digested in hot water, the fluid filtered, and again evaporated. The water

* I speak of the serosity in its entire state, as procured in the manner mentioned in the beginning of this paper. When a considerable part of it has been evaporated, the residue I have found to be alkaline. We must therefore suppose, that there exists in serosity a very minute quantity of alkali, but commonly too little to be detected by turmeric: it must be less than $\frac{1}{200}$ of its weight, considerably less than that in the serum from which the serosity is procured. 
was tinged brown, and as the evaporation proceeded, evidently shewed that it contained a considerable proportion of animal matter. At length small cubical crystals were formed, which, when purified by a subsequent solution and crystallization, were found to be nearly in the proportion of $1 \frac{1}{2}$ grain in one ounce of the entire serum.

The most remarkable and characteristic property of albumen is its coagulation by heat, and by different chemical substances, particularly by alcohol and by acids. No explanation of this phenomenon has been offered which appears to me satisfactory, nor indeed does it seem easy to refer the operation of such different agents to any one principle. The hypothesis, which is by far the most ingenious, and which, from the authority of its supporters, claims every attention, was originally proposed by Dr. Thompson *, and afterwards more fully developed by Mr. Brandet. Mr. Brande adopted it in consequence of his discovery, that the negative extremity of the Galvanic apparatus has the power of coagulating albumen, an effect which he explains upon the principle, that the action consists simply in the abstraction of an alkali, which was before in combination with the albumen, and which preserved it in the liquid state. Uncoagulated albumen he therefore considers as essentially an alkaline solution, and that heat acts by causing some new disposition of the affinities, in

* Chemistry, v. 489. + Phil. Trans. 1809. p. 373. 
consequence of which the alkali is transferred from the albumen to the water. Alcohol and the acids, he supposes, in like manner, produce their effect by combining with the alkali. But to this hypothesis there are, I conceive, insuperable objections. In the first place, although it is admitted that alkalies possess the property of dissolving albumen, yet for this purpose a quantity is necessary, very much greater than what we have reason to suppose exists in serum. If the affinities are so nicely balanced in the serum, that the mere application of heat can separate the alkali from the albumen, much more would it permit itself to be displaced by an acid, so that when a quantity of acid has been added, sufficient to produce an excess of acidity in the serum, we must conclude, that all the alkali has been neutralized. The above experiments shew how very minute this quantity of alkali is, and how totally inadequate to produce the effects assigned to it. That the mere removal of the alkali from the albumen does not cause its coagulation, I think may be concluded from the fact, that acetic acid may be added to the albumen in more than 100 times the quantity necessary for saturation, without any appearance of coagulation taking place. This seems to prove; that coagulation does not depend merely upon the abstraction of the alkali, and, I confess, appears to me quite decisive against the hypothesis of Mr. Brande. At the same time I acknowledge myself unable to substitute any other in its place, and therefore I content myself at present with expressing the fact, 
that the action of heat upon albumen is specific, unlike any other phenomenon, with which we are acquainted, and not capable of being referred to any general principle.

The operation of alcohol upon albumen I conceive to depend, in a great measure at least, upon its power of abstracting water from it. This opinion I ground upon the following circumstances : when albumen is only moderately diluted, the effect of this re-agent is greatly diminished, or even altogether destroyed. If we dilute serum with six times its bulk of water, the effect of alcohol is scarcely perceptible, while oxymuriate of mercury converts the fluid into a thick cream. If alcohol be poured into serum, its superior levity keeps it on the surface, and it immediately produces a coagulation in the upper stratum of the albumen; but if a quantity of water be added, the coagulum is instantly removed; and the same may be effected by exposing it to a gentle heat, when the alcohol is evaporated, and the serum is left in the same state as before the experiment. This circumstance proves that albumen, as coagulated by heat, is a very different substance from the coagulum that is formed by alcohol, and from this difference in the result we may infer that the processes themselves are different in their nature.

The coagulation of albumen by sulphuric acid must, I conceive, likewise be referred, in some 
measure, to its affinity for water, because in this case, as in that of alcohol, if the serum be comsiderably diluted, it quickly loses the property of being coagulated by the acid, and a coagulum which has been formed may be re-dissolved, by the addition of a considerable quantity of water. If the serum be diluted with six times its bulk of water, no immediate effect takes place from the addition of sulphuric acid, and after serum has been coagulated by sulphuric acid, if a quantity of water be immediately added, and the mixture agitated, the coagulum is entirely re-dissolved, and the fluid is rendered transparent; but although the first effect of the acid be owing to the sudden abstraction of the watery part of the serum, yet it seems that afterwards a union takes place between the acid and the albumen, for even in serum that is considerably diluted, a coagulum is gradually produced which is insoluble.

The oxymuriate of mercury has been classed among the substances which coagulate albumen; yet, strictly speaking, I should regard it rather as a precipitant than as a coagulator. It enters into a chemical union with albumen, and forms an insoluble substance which subsides from the fluid; it even acts so powerfully, as that when it is added to albumen, and heat is afterwards applied, it deatroys the coagulum which would otherwise be formed, and entirely separates the water from the solid part of the serum. The following experiment VOL. 1. 
is, I think, a decided proof that the oxymuriate of mercury forms a real chemical union with albumen. The oxymuriate was added to serum in sach proportion as to separate all the albumen, and to leave the fluid transparent; an equal bulk of water had the same quantity of oxymuriate added, and to equal measures of this solution, and of the fluid from the former experiment, the muriate of tin was added; in the fluid a turbidness was produced, and a small grey precipitate slowly subsided; in the solution a copious black precipitate was formed, which fell to the bottom in considerable quantity. An experiment of a similar kind was made with pure potash, by adding it in equal quantities to the solution of the oxymuriate of mercury, and to the fluid that was separated from a mixture of oxymuriate and serum. Although there was some variety as to the effects produced on different specimens of serum by the oxymuriate of mercury, yet generally it appeared, that if to a quantity of serum half its weight of a saturated solution of the oxymuriate of mercury was added, and the mixture boiled, the albumen was all separated, the fluid was left transparent, and was affected neither by the oxymuriate nor by serum, shewing that they had mutually saturated each other. The same circumstance, however, seemed to take place here, which had formerly been observed with respect to the compound of tan and jelly, that although there is one particular proportion in which the substances, seem most disposed to unite, yet according to the 
quantities of each that are added together, a considerable variation is produced in the nature of the product. In a comparative experiment, which was made for the purpose of ascertaining this point, it was found, that when the same quantity of the oxymuriate of mercury was added to two portions of diluted serum, one containing only half as much albumen as the other, the weights of the precipitates were as 9 to 15 . When dried by a moderate heat, the precipitate which contained the greater proportion of the oxymuriate was harder, and of a blacker colour than the other*.

The following conclusions may be deduced from the experiments that are detailed in this paper: 1. The serosity of the blood contains no jelly. 2. It contains a minute quantity of albumen. 3. It contains about 2 per cent. of solid contents; the chief part of which is an animal matter different both from jelly and albumen. 4. It contains a little muriate of soda, and probably also a minute quantity of uncombined alkali. 5. The animal matter peculiar to serosity is not affected either by the oxymuriate of mercury or by tan, and it is not, like albumen, rendered insoluble by heat. 6 . The

* The effect of the oxymuriate of mercury in coagulating serum appears to have been first discovered by Boyle. In the "Natural History of the blood," he relates the following experiment : "To " try also what a salt compounded with a metal would do upon " our serum, we put to it a little strong solution of sublimate " with which it presently afforded a white and curdy substance." 
tpecific gravity of serum is generally at more than 1.023. 7. Its solid contents are generally sbout 12 per cent. 8. The quantity of ancombined atkali in one ounce of serum is cotturated by one grain of sulphuric acid. 9. The alkati is in the caustic state. 10. Serum contains abont rto of its weight of the muriate of soda. 11. It is probable the coagulation of atbumen by heat does not depend upon any chemical change in the relation of the alkali to the albumen. 12. It is probable that alcohol coagulates albumen principally by the sudden abstraction of water from it. 13. Sulptruric acid acts partly on the same principle, but pertly also from a chemical union between the acid and the albumen. 14. The oxymuriate of mercury acts by forming a chemical compound with the albumen which is insoluble in water. 15. The compound of the oxymuriate of mereury and albamen is not uniform in the proportion of its constituents. 